\title{
Detection of Isolated Metal Ions on Ferritin by Single-Particle Cryo-STEM Reconstruction
}

\author{
Nadav Elad ${ }^{1}$, Giuliano Bellapadrona ${ }^{2}$, Lothar Houben ${ }^{1}$, Irit Sagi ${ }^{3}$, Michael Elbaum $^{2}$
}

[1] Dept of Chemical Research Support, Weizmann Institute of Science, Rehovot, Israel.

[2] Dept of Materials and Interfaces, Weizmann Institute of Science, Rehovot, Israel.

[3] Dept of Biological Regulation, Weizmann Institute of Science, Rehovot, Israel.

Observation of single metal atoms was one of the first applications of annular dark field scanning transmission electron microscopy (ADF-STEM) [1]. Heavy atoms on a lighter background, such as metal ions on protein structure, could in principle be detected by stronger scattering of the heavy atom to high angles, i.e., by atomic number or " $Z$ " contrast. Cryo-microscopy imposes severe constraints on electron exposure, however, so the prospect for detection of single atoms by STEM is uncertain. In conventional phase contrast cryoTEM, spherical aberration and defocus combine to weaken the signal of isolated heavy elements metal ions bound to proteins or other macromolecules. Metal atoms can be identified only when the protein structure can be reconstructed to high resolution. STEM imaging is performed in focus, and, absent an image forming lens, is relatively immune to both spherical and chromatic aberrations. We asked whether the scattering signal is sufficiently strong to detect single metal atoms by $\mathrm{Z}$ contrast.

Ferritin is a well-known iron storage protein found in all kingdoms of life. Composed of 24 polypeptide chains, the protein forms a nearly-spherical assembly with octagonal (432) symmetry and a hollow core. Soluble iron enters through channels as Fe(II), undergoes oxidation to $\mathrm{Fe}(\mathrm{III})$ at specific ferroxidase motifs on the inner surface of the protein shell, and deposits in mineral form within the cavity. Key sites in the protein structure include: the channels, the ferroxidase sites behind the three-fold channels, and the putative mineral nucleation sites at the two-fold axes. Notably, zinc binds to the ferroxidase site more strongly than iron and prevents further filling with metal ions.

We demonstrate here the detection of isolated atoms of $\mathrm{Zn}$ and $\mathrm{Fe}$ on ferritin using cryogenic ADF-STEM. Bacterially expressed human heavy-chain ferritin was incubated with metal salts at a stoichiometry of 48:1 for Zn, two ions per protein subunit, or at 100:1 for Fe. After single-particle reconstruction, $\mathrm{Zn}$ is found in a pattern that matches precisely its location at the ferroxidase sites determined previously by X-ray crystallography [2]. By contrast, the Fe distribution is smeared along an arc corresponding to the proposed path from the ferroxidase sites to the mineral nucleation sites along the two-fold axes. In this case the single-particle reconstruction is interpreted as a probability distribution based on the ensemble average of individual locations.

The results demonstrate atomic detection of isolated metal atoms in biomolecules and provide a practical measure of their visibility vis a vis the protein background. Detection of isolated metal atoms by cryo-STEM imaging opens new horizons for electron cryo-microscopy and tomography. 


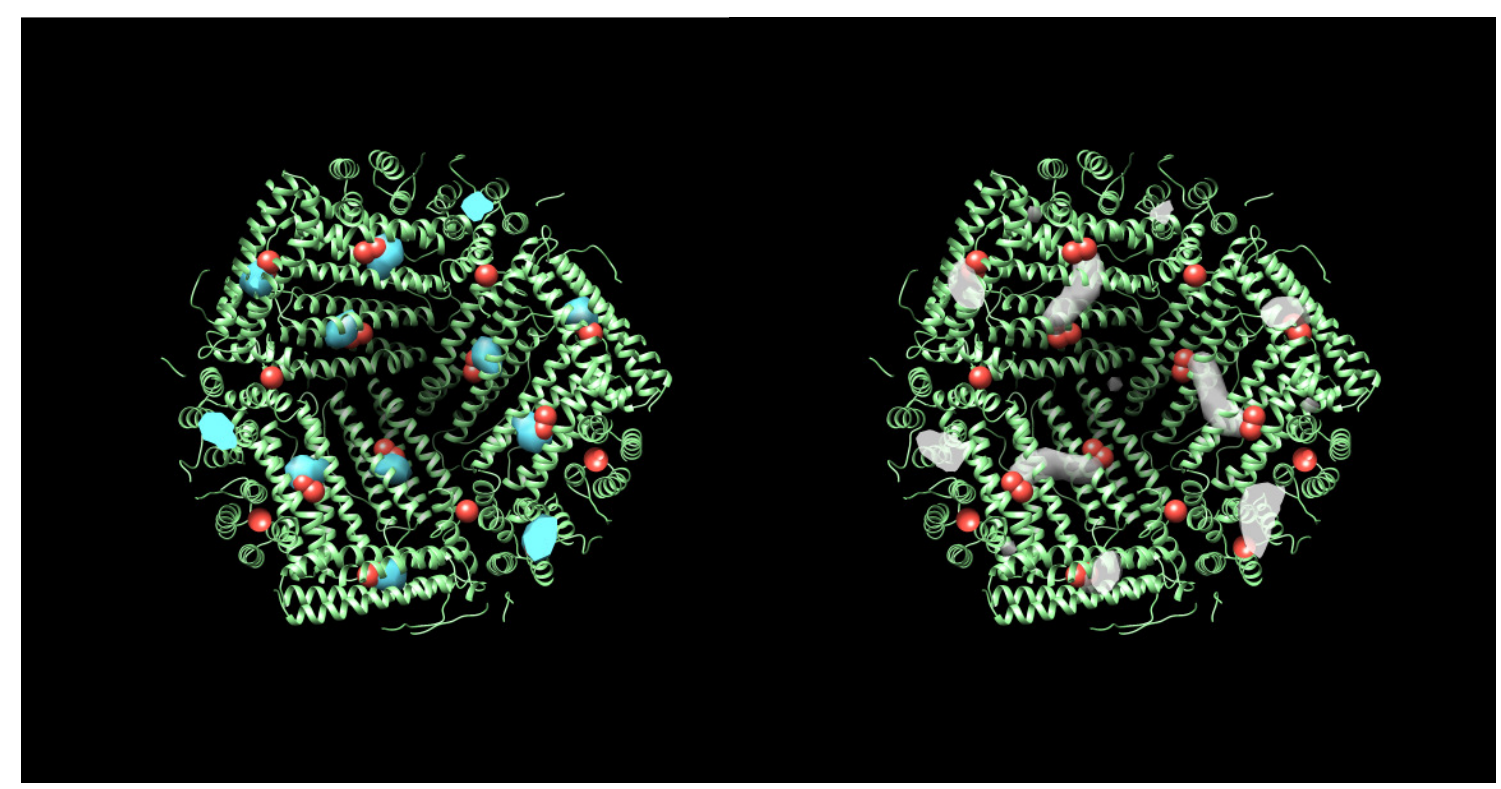

Figure 1. The inner surface of the human heavy-chain ferritin structure (PDB: 2CEI) is represented by the green ribbon diagram, on which the Zn-labelled ferroxidase sites are shown in red [2]. In the present work, bacterially-expressed protein was incubated either in $\mathrm{Zn}$ or Fe solution at stoichiometry of 48 or 100:1, metal:protein, respectively. In the case of $\mathrm{Zn}$, a strong scattering signal (blue) corresponds to the ferroxidase sites identified in the crystal structure. Fe (transparent gray), by contrast, induces scattering in an arc spanning adjacent ferroxidase sites, with an additional density at the three-fold channel.

[1] AV Crewe, J Wall, J Langmore, Visibility of Single Atoms. Science. 168 (1970), p. 1338.

[2] L Toussaint, L Bertrand, L Hue, RR Crichton, J-P Declercq, High-resolution X-ray Structures of Human Apoferritin H-chain Mutants Correlated with Their Activity and Metal-binding Sites. J. Mol. Biol. 365 (2007), p. 440. 